\title{
TOPOLOGICALLY RIGID NON-HAKEN 3-MANIFOLDS
}

\author{
J. HASS and W. MENASCO
}

(Received 8 June 1992)

Communicated by J. H. Rubinstein

\begin{abstract}
A closed irreducible 3-manifold $M$ is topologically rigid if any homotopy equivalent irreducible 3-manifold is homeomorphic to $M$. A construction is given which produces infinitely many non-Haken topologically rigid 3-manifolds
\end{abstract}

1991 Mathematics subject classification (Amer. Math. Soc.): 57 N 10.

\section{Introduction}

A closed irreducible 3-manifold $M$ is topologically rigid if any homotopyequivalent irreducible 3-manifold is homeomorphic to $M$. It is conjectured that any closed irreducible 3-manifold with infinite fundamental group is topologically rigid. Waldhausen [13] proved this for Haken 3-manifolds. Scott showed that all Seifert fiber spaces with infinite fundamental group are topologically rigid [11]. If $M$ and $M^{\prime}$ are homotopy equivalent hyperbolic 3-manifolds, then the Mostow rigidity theorem implies they are homeomorphic [10], but if only $M$ is known to be hyperbolic then the question is open. Recently it was shown in [5] that an irreducible 3-manifold which contains an immersed surface satisfying the 1-line and 4-plane properties is topologically rigid. Various constructions are described there of 3-manifolds containing such surfaces. Non-Haken manifolds are probably generic in these constructions, but explicit non-Haken examples

(c) 1993 Australian Mathematical Society 0263-6115/93 \$A2.00+0.00

The first author was partially supported by NSF grants and the Sloan Foundation and the second author was partially supported by NSF grants and an AMS Fellowship. 
were not given in [5].

Aitchison and Rubinstein have studied geometrically cubed 3-manifolds, which contain immersed surfaces satisfying the conditions of [5], and shown that such surfaces persist under most surgeries on certain links [2]. In this paper we combine their ideas with an explicit calculation to construct the first examples of non-Haken, non-Siefert fibered 3-manifolds which are topologically rigid. We produce infinitely many such manifolds.

The first step is to construct a link $L$ in $S^{3}$ whose complement admits a complete hyperbolic structure which is the union of two regular ideal hyperbolic cubes. Next we show that $S^{3}-L$ contains no closed incompressible surfaces other than the peripheral tori. This is done using ideas developed in $[8,9]$ for classifying incompressible surfaces in link complements. Next we use results of Hatcher [6] and Gromov-Thurston [4] to show that infinitely many surgeries on this link give non-Haken negatively curved 3-manifolds. Finally we use ideas of [2] to show that these manifolds contain immersed surfaces with the 1-line and 4-plane properties, and so are topologically rigid by [5].

The paper is organized as follows. In Section 2 we exhibit a 3-component link $L$ in $S^{3}$ whose complement is the union of two hyperbolic cubes. In Section 3 we show that the complement of $L$ contains no closed incompressible surfaces. In Section 4 we show that infinitely many surgeries on $L$ give manifolds which contain immersed surfaces with the 1-line and 4-plane properties.

\section{A link $L$ whose complement is a union of two ideal hyperbolic cubes}

We consider the following 3-component alternating link $L$ in $S^{3}$ (see Figure 2.1).

Let $M$ denote the complement of $L$ in the 3 -sphere. $M$ has a a natural decomposition into two ideal cubes, one above and one below the projection plane. This decomposition is similar to the decomposition of the figure eight knot, the Borromean rings and other examples in [12]. See [7] for a general construction. The choice of $L$ was inspired by similar examples in [2], but those link complements do contain closed incompressible surfaces.

In this decomposition the two cubes are identified so that after the identifications there are six faces meeting each edge, and a total of three vertices, four edges and six faces. If the vertices are deleted and the two cubes are taken to be regular ideal cubes, then this gluing is realized by hyperbolic isometries, showing that $M$ has a complete hyperbolic structure. 


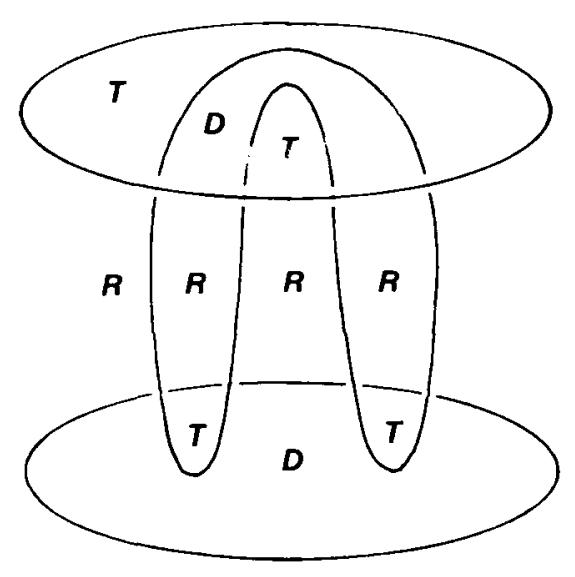

FIGURE 2.1

\section{The complement of $L$ contains no closed incompressible surfaces}

We wish to understand how incompressible surfaces can be embedded in $\boldsymbol{M}$. To do so we study pairwise incompressible surfaces, incompressible surfaces whose boundaries consist of curves parallel to a meridian of $L$ and for which any curve parallel in $M$ to a meridian of $\partial M$ is boundary parallel in the surface. Any closed incompressible surface in $S^{3}-L$ becomes pairwise incompressible after a series of surgeries along disks intersecting $L$ in one point. We study such surfaces via the techniques developed in [8]. We position $L$ in $S^{3}$ so that it lies on the union of two 2-spheres, $S_{+}^{2}$ and $S_{-}^{2}$. These agree except in a bubble around each crossing, where $S_{+}^{2}$ goes over the top hemisphere and $S_{-}^{2}$ goes over the bottom hemisphere. We can assume that $F$ intersects $L, S_{+}^{2}$ and $S_{-}^{2}$ transversely and that $F$ meets the bubbles in saddles, as in [8]. We will classify all possibilities for $F \cap S_{+}^{2}$.

$F \cap S_{+}^{2}$ consists of a collection of curves, meeting $L$ in punctures at boundary components of $F . L \cap S_{+}^{2}$ and the bubbles together cut $S_{+}^{2}$ into components which we call regions. These are the complementary components of $L$ in $S_{+}^{2} \cap S_{-}^{2}$. A curve of $F \cap S_{+}^{2}$ enters and leaves a region through a puncture or a bubble. Possibly there are some components of $F \cap S_{+}^{2}$ contained entirely in a region. Let $|F|$ denote the sum of the number of saddles, punctures and the number of components contained in a region. We isotop $F$ to minimize $|F|$. We then have 


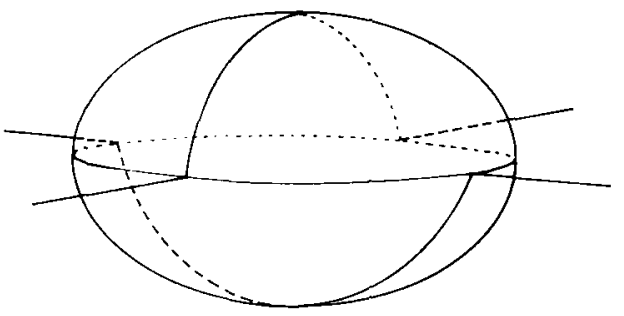

FIGURE 3.1

LEMMA 3.2. Let $F$ be a pairwise-incompressible surface in the complement of an alternating link $L$, with $|F|$ minimal in its isotopy class. Then either $F$ is a boundary parallel annulus or:

(i) Each curve in $F \cap S_{+}^{2}$ bounds a disk in $B_{+}^{3}$, the 3-ball in $S^{3}-L$ that $S_{+}^{2}$ bounds.

(ii) No component of $F \cap S_{+}^{2}$ is contained in a region.

(iii) A component of $F \cap S_{+}^{2}$ does not meet a bubble in more than one arc.

(iv) An arc of $F \cap S_{+}^{2}$ in a given region which meets a bubble is disjoint from the two boundary arcs of the region meeting that bubble.

(v) A curve in $F \cap S_{+}^{2}$ does not meet an arc in the boundary of a region in more than one point.

PROOF. Assume that $F$ is not a boundary parallel annulus.

(i) This follows from incompressibility of $F$.

(ii) If there is a component of $F \cap S_{+}^{2}$ with no saddles or punctures, take an innermost such. It bounds a disk in $B_{+}^{3}$, so we can isotop across it to reduce $|F|$.

(iii) If it does, we can reduce $|F|$ as in [8].

(iv) If it does, we can reduce $|F|$ as in [9].

(v) If it does, then $F$ is boundary compressible and hence compressible, since $\partial M$ is union of tori and $F$ is not an annulus.

There are three types of regions we need to study, as shown in Figure 2.1.

(1) Two-sided regions which we label $T$. There are four of these.

(2) Four-sided regions which meet $T$ regions on two sides. We label these $D$. There are two of these.

(3) Four-sided regions which meet $T$ regions on one side and $D$ regions on an opposite side. We label these $R$. There are four $R$ regions.

We now analyze all of the different possible ways that $F \cap S_{+}^{2}$ can cut through 
these regions for $F$ pairwise incompressible. We then piece together the different possibilities to construct all possible $F$ 's.

LEMMA 3.3. The only pairwise-incompressible surface in $M$ is a 4-punctured sphere passing once through each $R$ region. There are no closed incompressible surfaces in $M$ other than the peripheral torus.

ProOF. Let $F$ be an incompressible, pairwise-incompressible surface with $|F|$ minimal.

STEP 1 . We can isotop $F$ so that its intersection with a $T$ region consists of $n$ arcs passing through each of the two bubbles in $T, n \geq 0$. See Figure 3.4.

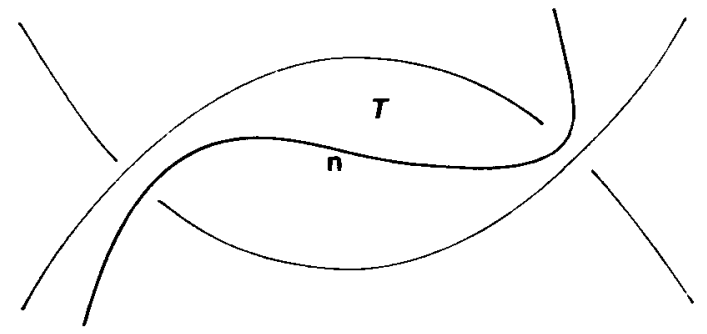

FIGURE 3.4. The configuration in a $T$ region.

PROOF OF STEP 1. An arc with a puncture passing through a bubble would violate (iv) of Lemma 3.2. An arc with two punctures would either violate (v) of Lemma 3.2, or would run between the two sides of $T$. In the latter case, we can isotop $F$ through a crossing so that it no longer meets $T$, as in Figure 3.5. This isotopy does not change $|F|$.

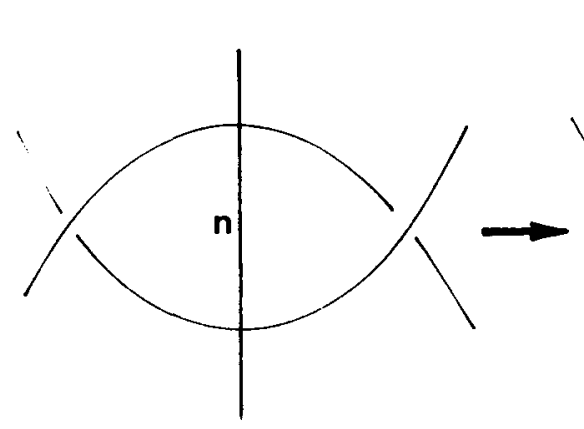

(a)

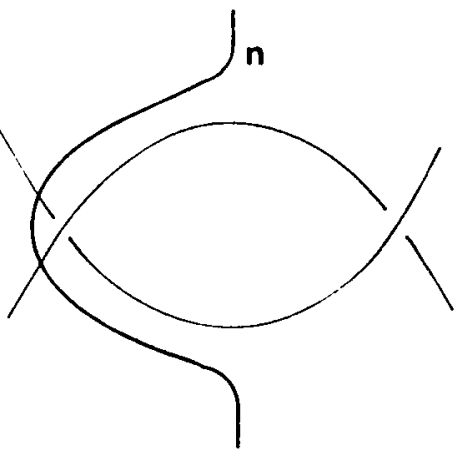

(b)

FIGURE 3.5 
STEP 2. The intersection of $F$ with a $D$ region consists of a finite number of arcs running between the two $R$ regions meeting it as in Figure 3.6a, or $n$ arcs passing through each of the saddles in the $D$ region, as in Figure 3.6b.

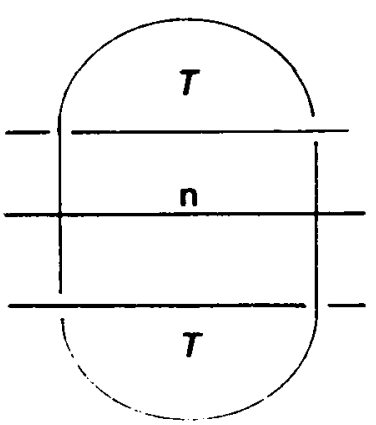

(a)

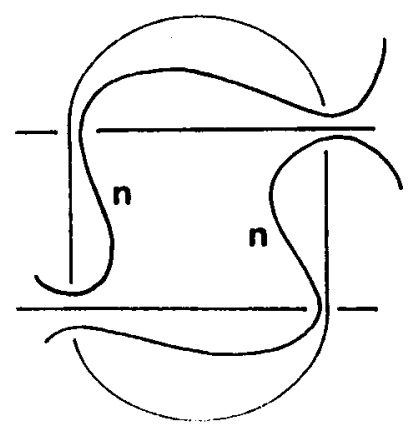

(b)

FIGURE 3.6. The possible configurations in a $D$ region.

PROOF OF STEP 2. If a saddle occurs at any one of the four crossings meeting $D$, then the picture for the adjacent $T$ region is given by Step 1, so that $n \operatorname{arcs}$ emerge from each of the bubbles meeting that $T$ region. None of these arcs can join up to one another by (iii) of Lemma 3.2. Neither can they leave $D$ in a puncture, by (iv) of Lemma 3.2. We conclude that they must emerge through the other two bubbles as in Figure 3.6b.

If no saddles occur, then the only possibility is $n$ arcs running from one of the $R$ regions meeting the $D$ region to the other one, since no punctures meet the adjacent $T$ regions by Step 1. This is shown in Figure 3.6a.

STEP 3. The intersection of $F$ with an $R$ region consists of a finite number of arcs running between the two adjacent $R$ regions, as in Figure 3.7a, or a finite number of arcs passing through each of the bubbles in the $R$ region, as in Figure 3.7b. 


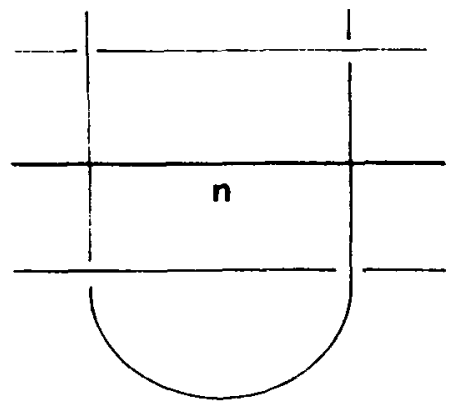

(a)

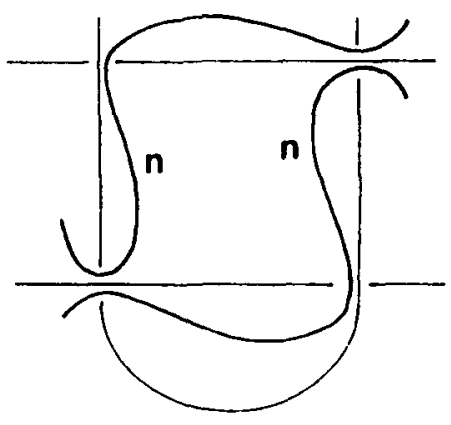

(b)

FIGURE 3.7. The possible configurations in an $R$ region.

PROOF OF STEP 3. If an arc enters the $R$ region through a bubble from the adjacent $D$ region then Step 2 implies that the picture in that $D$ region is given by Figure 3.8a. The same argument that applied in Step 2 now shows that the picture in the $R$ region is as in Figure 3.8b.

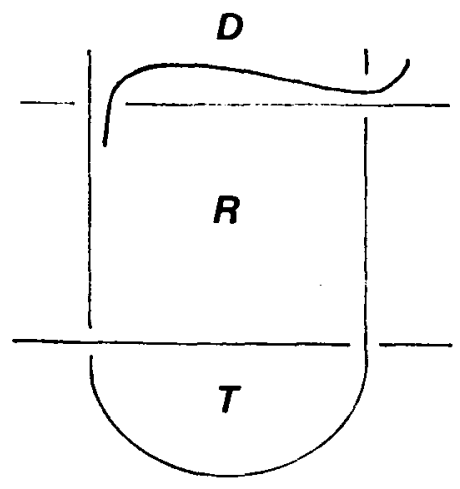

( a)

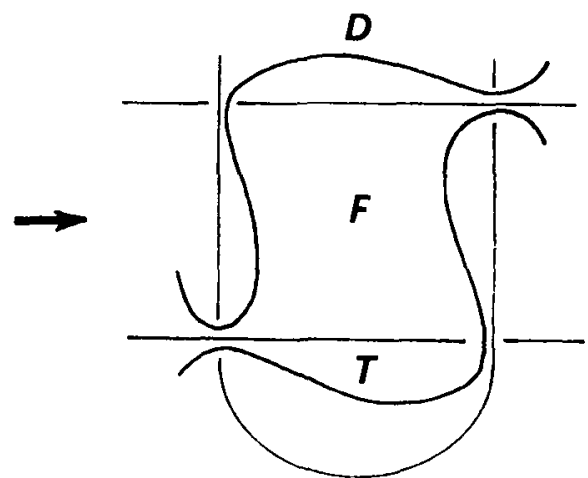

(b)

FIGURE 3.8

If the arc enters through a bubble on the $T$ side, as in Figure 3.9a, then we consider the adjacent $R$ region, where an arc enters through a bubble on the $D$ side. The picture in the adjacent $R$ region is determined by the previous 
argument, implying that an arc also enters the original $R$ region from a bubble meeting the adjacent $D$ region, and the original $R$ region is as in Figure 3.7b.

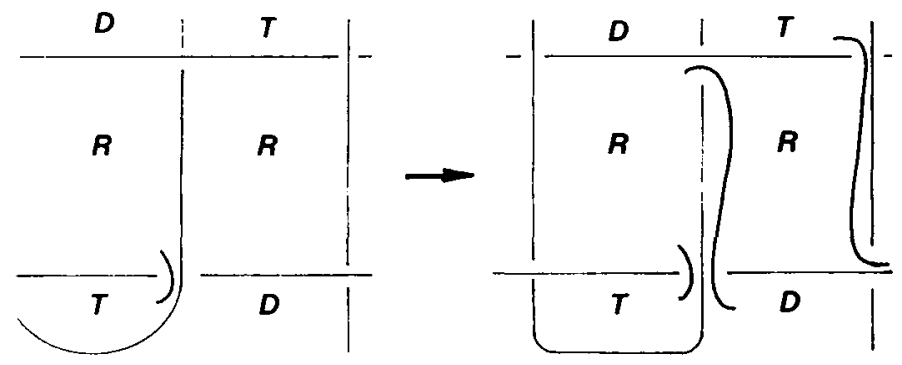

(a) (b)

FIGURE 3.9

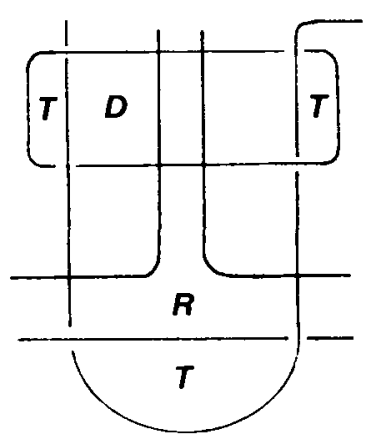

(a)

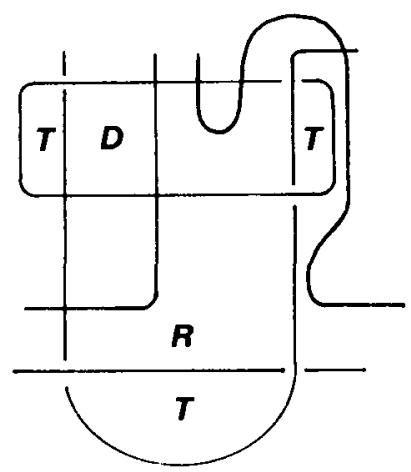

(b)

FIGURE 3.10

The remaining possibility is that there are no saddles in the $R$ region. Either we are in the case of Figure 3.7a, as desired, or the picture consists of arcs of the type shown in Figure 3.10a. The picture in the adjacent $T$ and $D$ regions is then determined by Steps 1 and 2. If such arcs exist, we can isotop across a $T$ region to get a picture in the adjacent $D$ region which violates (v) of Lemma 3.2, allowing $|F|$ to be reduced and finishing Step 3.

Putting these pictures together, we get closed curves which have either only punctures or only saddles as they cross the link projection. The first case gives a collection of parallel four-punctured spheres cutting through each $R$ region once. The second possibility gives rise to curves meeting a single saddle twice, 
violating (iii) of Lemma 3.2, and so is impossible.

Suppose we tube together the boundary components of a collection of parallel 4-punctured spheres to get a closed incompressible surface. All the boundary components of the punctured spheres are parallel curves on a peripheral torus of one of the components of $L$. Therefore some tube runs between curves which are adjacent on the peripheral torus. If this tube connects two parallel 4-punctured spheres then the region between them is compressible, irrespective of where the other tubes run. If the tube runs from a punctured sphere to itself, as in Figure 3.11, then the resulting punctured torus is compressible, irrespective of where the other tubes are located. In Figure 3.11 there is a compressing disk for the twice-punctured torus running twice over the tube. The boundary of the disk is shown as a dashed line. We conclude that there are no non-trivial closed incompressible surfaces in the link complement.

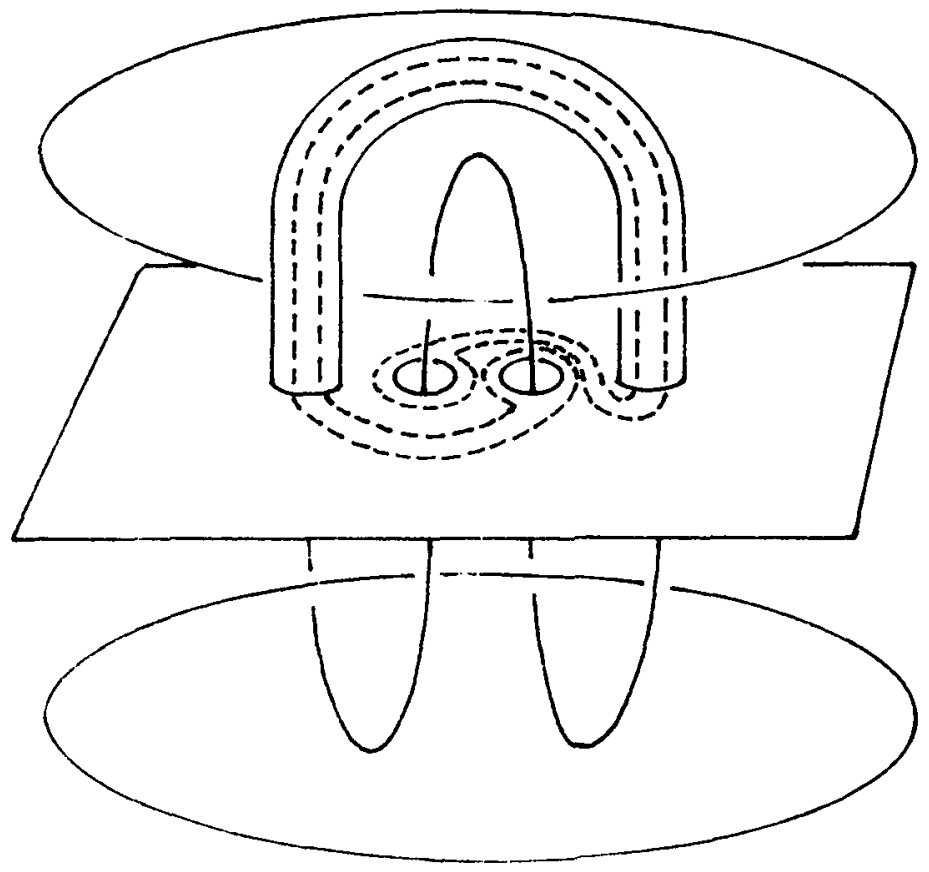

FIGURE 3.11 


\section{Construction of topologically rigid non-Haken 3-manifolds}

THEOREM 4.1. An infinite number of surgeries on $L$ give rise to topologically rigid non-Haken 3-manifolds.

PROOF. Simple curves on the peripheral tori of $M$, and thus surgeries on $L$, are parametrized by $\left(\mathbf{Z}^{2} / \pm\right)^{3}$. Projectivizing to identify a collection of parallel copies of a curve gives rise to the projective lamination space called $P L(\partial M)$, homeomorphic to $S^{5}[6]$. This is a completion of the space of surgeries, which form a dense subset. The rational points on $P L(\partial M)$ correspond to Dehn surgeries on $L$. A theorem of Hatcher states that the points in $P L(\partial M)$ which bound an incompressible, boundary incompressible surface in $M$ are contained in a two-dimensional submanifold of $S^{5}[6]$. Since $M$ contains no closed incompressible surfaces, and boundary compressible surfaces are either compressible or boundary parallel annuli, this implies that away from this two dimensional submanifold, Dehn surgeries give rise to closed non-Haken 3-manifolds.

Since the complement of $L$ has a complete hyperbolic structure, the $2 \pi$ Theorem of Gromov-Thurston $[3,4]$ implies that after finitely many surgery coefficients are excluded for each component, all surgeries on $L$ yield closed 3-manifolds of strictly negative curvature. The metrics on these manifolds agree with the hyperbolic metric on $S^{3}-L$ in an arbitrarily large compact subset. Thus, given a compact subset $K$ of the link complement, there is a codimension one submanifold of $P L(\partial M)$ such that all surgeries on $L$ which miss this submanifold yield closed negatively curved 3-manifolds with constant hyperbolic metric on a neighborhood of $K$. Since the union of the two exceptional submanifolds just described has positive codimension, infinitely many surgeries on $L$ give non-Haken, negatively curved 3-manifolds, with the same hyperbolic metric on $K$.

The complement of $L$ in these closed negatively curved manifolds is the union of two cubes with deleted vertices. Consider the immersed surface $G$ constructed by taking the union of the six bisecting squares in the two cubes. In the complete hyperbolic 3-manifold $M, G$ is a totally geodesic immersed surface which has angles $\pi / 2$ along all self-intersections. As observed in [5], this implies that $G$ has the 1-line and 4-plane properties. Take $K$ to be the image of $G$ in $S^{3}-L$. Then for large surgery coefficients the manifolds obtained by surgery on $L$ have metrics of non-positive curvature which agree with the metric of $M$ in a neighborhood of $G$. Thus these manifolds have a metric of non-positive curvature in which $G$ is a totally geodesic surface whose self- 
intersections consist of double curves where two sheets meet at right angles. The lift of $G$ to the universal cover of one of these negatively curved manifolds consists of a collection of embedded totally geodesic planes which intersect orthogonally. It follows immediately that $G$ is $\pi_{1}$-injective. $G$ satisfies the 1-line property, since two totally geodesic planes can intersect in at most one line by the uniqueness of geodesic arcs connecting two points in a simply connected negatively curved manifold. It remains to check that $G$ satisfies the 4-plane property. Note first that if three planes meet pairwise then they have a common point, as otherwise we could form a geodesic triangle with all right angles, impossible in a negatively curved space. Suppose now that we have four planes, all meeting pairwise. Looking at one of these planes, the three lines of intersection with the other three planes form an all right triangle on this plane, impossible since its induced curvature is strictly negative. Thus $G$ satisfies the 4-plane property in any of these manifolds.

Combining the above results we see that an infinite number of surgeries on $L$ give rise to closed, non-Haken 3-manifolds that contain surfaces satisfying the 1-line and 4-plane properties. The principal result of [5] is that such 3-manifolds are topologically rigid, concluding the proof of Theorem 4.1 .

\section{Acknowledgements}

We would like to thank Hyam Rubinstein for relating to us the beautiful constructions of cubed 3-manifolds in [2] which motivated this work. The first author would also like to thank the Institute for Advanced Study, where he was visiting during the course of this work. Both authors were partially supported by NSF grants.

\section{References}

[1] I. R. Aitchison, E. Lumsden and J. H. Rubinstein, 'Cusp structure of alternating links', Invent. Math., to appear.

[2] I. R. Aitchison and J. H. Rubinstein, 'An introduction to polyhedral metrics of non-positive curvature on 3-manifolds', preprint.

[3] S. Bleiler and C. Hodgson, 'Spherical space forms and Dehn surgery', preprint.

[4] M. Gromov and W. Thurston, 'Pinching constants for hyperbolic manifolds', Invent. Math. 89 (1987), 1-12. 
[5] J. Hass and P. Scott, 'Homotopy equivalence and homeomorphism of 3-manifolds', Topo$\log y 31$ (1992), 493-517.

[6] A. E. Hatcher, 'On the boundary curves of incompressible surfaces', Pacific J. Math. 99 (1982), 373-377.

[7] W. Menasco, 'Polyhedral representation of link complements', Contemp. Math. 20 (1983), 305-325.

[8] —, 'Closed incompressible surfaces in alternating knot and link complements', Topology 23 (1984), 37-44.

[9] — 'Determining incompressibility of surfaces in alternating knot and link complements', Pacific J. Math. 117 (1985), 353-370.

[10] G. D. Mostow, 'Quasi-conformal mappings in $n$-space and the rigidity of hyperbolic space forms', Inst. Hautes Études Sci. Publ. Math. 34 (1968), 53-104.

[11] P. Scott, 'There are no fake Seifert fiber spaces', Ann. of Math. 117 (1983), 35-70.

[12] W. Thurston, The geometry and topology of 3-manifolds (Lecture notes, Princeton University, 1978).

[13] F. Waldhausen, 'On irreducible 3-manifolds which are sufficiently large', Ann. of Math. 87 (1968), 56-88.

University of California

SUNY at Buffalo

Davis, California

Buffalo, New York

U.S.A.

U.S.A.

e-mail: jhass@ucdavis.edu

e-mail: mthtwwm@ubvmsc.cc.buffalo.edu 